\title{
New Medications for the Treatment of Diabetes
}

\author{
Satish K. Garg and Dominique Giordano
}

Introduction

A S IN THE PREVIOUS DECADE, the number of new medications and/or their newer $A$ indications for the treatment of patients with diabetes have continued to evolve in the last year. Many manuscripts last year highlighted the importance of adjunctive therapeutic options for patients with type 1 diabetes (T1D), where most of the patients are not able to achieve target hemoglobin A1c (HbA1c) levels. We will discuss in detail the role of sodium-glucose cotransporter 2 (SGLT2) inhibitors in the management of T1D.

There continues to be a debate as to whether insulin degludec or U300 glargine is better, but we are assuming this will be discussed in the insulin section of this yearbook. We will briefly discuss the biosimilar rapid-acting and long-acting insulin analogs, as the cost of insulin in the United States continues to be a problem. The longer-acting glucagonlike peptide (GLP) analogs (weekly injections of semaglutide) show better efficacy than the existing daily GLP analogs (liraglutide) or weekly GLP-1 analogs (dulaglutide). The side effects noted in the risk of increased diabetic retinopathy with semaglutide were probably related to a rapid decline in HbA1c values in patients with type 2 diabetes (T2D), and thus, no special label change was recommended when the drug was approved by the U.S. Food and Drug Administration (FDA) earlier in 2018. As one can imagine, it is difficult to cover all of the emerging therapies in the field of diabetes, and thus we have chosen the following topics that might be of interest to the readers.

Key Articles Reviewed for the Article
Effects of sotagliflozin added to insulin in patients with type 1 diabetes
Garg SK, Henry RR, Banks P, Buse JB, Davies MJ, Fulcher GR, Pozzilli P, Gesty-Palmer D,
Lapuerta P, Simó R, Danne T, McGuire DK, Kushner JA, Peters A, Strumph P
$N$ Engl J Med 2017; 377: 2337-2348
Sotagliflozin in combination with optimized insulin therapy in adults with type 1
diabetes: the North American inTandem1 study
Buse JB, Garg SK, Rosenstock J, Bailey TS, Banks P, Bode BW, Danne T, Kushner JA,
Lane WS, Lapuerta P, McGuire DK, Peters AL, Reed J, Sawhney Strumph P
Diabetes Care 2018; 41: $1970-1980$
HbA1c and hypoglycemia reductions at 24 and 52 weeks with sotagliflozin in
combination with insulin in adults with type 1 diabetes: the European inTandem2
study
Danne T, Cariou B, Banks P, Brandle M, Brath H, Franek E, Kushner JA, Lapuerta P,
McGuire DK, Peters AL, Sawhney S, Strumph P
Diabetes Care 2018; 41: 1981-1990

Barbara Davis Center for Diabetes, University of Colorado, Denver, CO 
Efficacy and safety of dapagliflozin in patients with inadequately controlled type 1 diabetes (DEPICT-1): 24 week results from a multicentre, double-blind, phase 3 , randomised controlled trial

Dandona P, Mathieu C, Phillip M, Hansen L, Griffen SC, Tschöpe D, Thorén $F, X u J$, Langkilde AM; DEPICT-1 Investigators

Lancet Diabetes Endocrinol 2017; 5: 864-876

Cardiovascular mortality and morbidity in patients with type 2 diabetes following initiation of sodium-glucose co-transporter-2 inhibitors versus other glucose-lowering drugs (CVD-REAL Nordic): a multinational observational analysis

Birkeland KI, Jorgensen ME, Carstensen B, Persson F, Gulseth HL, Thuresson M, Fenici P, Nathanson D, Nyström T, Eriksson JW, Bodegård J, Norhammar A

Lancet Diabetes Endocrinol 2017; 5: 709-717

Canagliflozin in conjunction with sulfonylurea maintains glycemic control and weight loss over 52 weeks: a randomized, controlled trial in patients with type 2 diabetes mellitus

Yale JF, Xie J, Sherman SE, Garceau C

Clin Ther 2017; 39: 2230-2242

Dapagliflozin/saxagliptin fixed-dose tablets: a new sodium-glucose cotransporter 2 and dipeptidyl peptidase 4 combination for the treatment of type 2 diabetes

Coppenrath VA, Hydery $T$

Ann Pharmacother 2018; 52: 78-85

Sodium glucose co-transporter 2 inhibitor luseogliflozin in the management of type 2 diabetes: a drug safety evaluation

Yabe D, Hamamoto Y, Seino Y, Kuwata H, Kurose T, Seino $Y$

Expert Opin Drug Saf 2017; 16: 1211-1218

Effects of sodium glucose cotransporter-2 inhibitors on serum uric acid in type 2 diabetes mellitus

Ahmadieh H, Azar $S$

Diabetes Technol Ther 2017; 19: 507-512

Altered patterns of early metabolic decompensation in type 1 diabetes during treatment with a SGLT2 inhibitor: an insulin pump suspension study

Patel NS, Van Name MA, Cengiz E, Carria LR, Weinzimer SA, Tamborlane WV, Sherr JL Diabetes Technol Ther 2017; 19: 618-622

Efficacy and Safety of Biosimilar SAR342434 insulin lispro in adults with type 1 diabetes also using insulin glargine-SORELLA 1 study

Garg SK, Wernicke-Panten K, Rojeski M, Pierre S, Kirchhein Y, Jedynasty $K$

Diabetes Technol Ther 2017; 19: 516-526

Efficacy and safety of MK-1293 insulin glargine compared with originator insulin glargine (Lantus) in type 1 diabetes: a randomized, open-label clinical trial

Home PD, Lam RLH, Carofano WL, Golm GT, Eldor R, Crutchlow MF, Marcos MC, Rosenstock J, Hollander PA, Gallwitz B

Diabetes Obes Metab 2018; 20: 2220-2228

Anti-insulin antibodies and adverse events with biosimilar insulin lispro compared with Humalog insulin lispro in people with diabetes

Home P, Derwahl KM, Ziemen M, Wernicke-Panten K, Pierre S, Kirchhein Y, Garg SK Diabetes Technol Ther 2018; 20: 160-170 


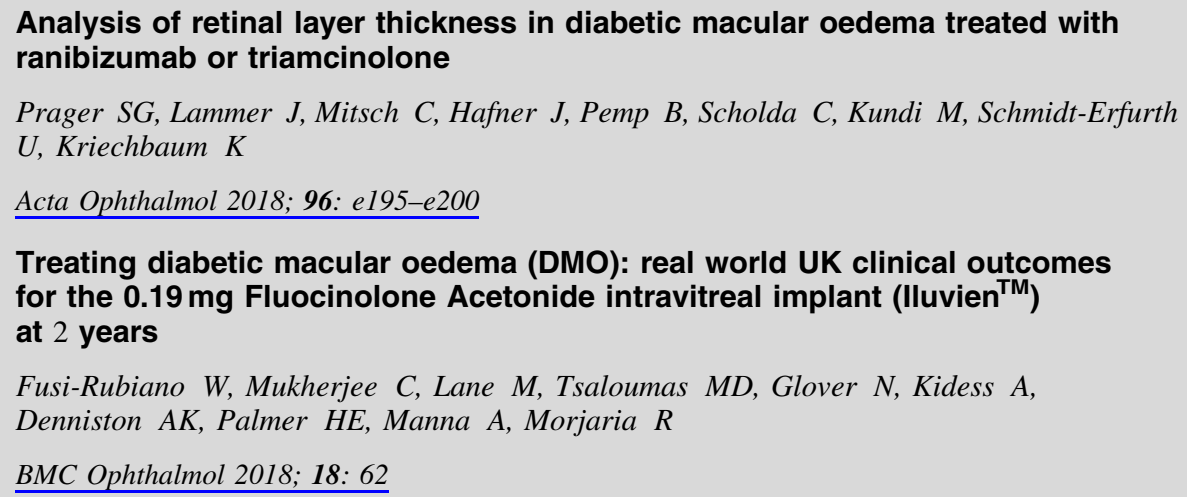

Fusi-Rubiano W, Mukherjee C, Lane M, Tsaloumas MD, Glover $N$, Kidess A, Denniston AK, Palmer HE, Manna A, Morjaria $R$

BMC Ophthalmol 2018; 18: 62

\section{Effects of sotagliflozin added to insulin in patients with type 1 diabetes}

Garg $\mathrm{SK}^{1}$, Henry $\mathrm{RR}^{2}$, Banks $\mathrm{P}^{3}$, Buse $\mathrm{JB}^{6}$, Davies $\mathrm{MJ}^{7}$ Fulcher $G^{8}{ }^{8}$, Pozzilli $P^{9}$, Gesty-Palmer $\mathrm{D}^{3}$, Lapuerta $\mathrm{P}^{3}$, Simó $\mathrm{R}^{10}$, Danne $T^{l l}$, McGuire $\mathrm{DK}^{4}$, Kushner $\mathrm{JA}^{5}$, Peters $A^{12}$, Strumph $\mathrm{P}^{3}$

${ }^{1}$ University of Colorado Denver, Aurora, CO; ${ }^{2}$ University of California at San Diego, San Diego, CA; ${ }^{3}$ Lexicon Pharmaceuticals, The Woodlands, TX; ${ }^{4}$ University of Texas Southwestern Medical Center, Dallas, TX; ${ }^{5}$ Baylor College of Medicine and Texas Children's Hospital, Houston. TX; ${ }^{6}$ Diabetes Research Center, University of North Carolina School of Medicine, Durham, NC; ${ }^{7}$ University of Leicester and University Hospitals of Leicester NHS Trust, Leicester, UK $;{ }^{8}$ University of Sydney, Sydney, Australia; ${ }^{9}$ University Campus Bio-Medico of Rome, Rome, Italy; ${ }^{10}$ Vall d'Hebron Research Institute, Barcelona, and CIBERDEM-Instituto de Salud Carlos III, Madrid, Spain; ${ }^{11}$ Diabetes Center Auf der Bult, Hannover Medical School, Hannover, Germany; ${ }^{12}$ University of Southern California, Los Angeles, CA

N Engl J Med 2017; 377: 2337-2348

\section{Background}

Most patients with type 1 diabetes (T1D) are unable to adequately control blood glucose levels with insulin therapy alone. Sotagliflozin is a new oral inhibitor of sodium-glucose cotransporters 1 and 2 that reduces postprandial hyperglycemia as well as reducing renal glucose absorption. This phase 3 trial evaluated the safety and effectiveness of sotagliflozin in combination with insulin treatment in patients with T1D.

\section{Methods}

A phase 3, double-blind, placebo-controlled trial of 1402 patients with T1D on insulin therapy (pump or injections) was conducted at 133 centers in 19 countries. Participants were randomly assigned to receive sotagliflozin $(400 \mathrm{mg}$ per day) or placebo in addition to their ongoing insulin therapy for 24 weeks. The primary endpoint was HbA1c level $<7.0 \%$ at week 24, with no episodes of severe hypoglycemia or diabetic ketoacidosis after randomization. Secondary endpoints were change from baseline to week 24 in $\mathrm{HbA1c}$, body weight, systolic blood pressure, and mean daily bolus dose of insulin.

\section{Results}

Compared with the placebo group, a higher proportion of patients in the sotagliflozin group achieved the primary endpoint (200 of 699 patients [28.6\%] vs 107 of 703 [15.2\%], $P<0.001)$. The sotagliflozin group had significantly greater least-squares mean change from baseline than the placebo group for HbA1c ( -0.46 percentage points), systolic blood pressure $(-3.5 \mathrm{~mm} \mathrm{Hg})$, weight $(-2.98 \mathrm{~kg})$, and mean daily bolus dose of insulin ( -2.8 units per day) $(P \leq 0.002$ for all comparisons). Both groups had a similar rate of severe hypoglycemia (3.0\% [21 patients] and 2.4\% [17] for sotagliflozin and placebo, respectively). The sotagliflozin group had a significantly lower rate of documented hypoglycemia with a blood glucose level of $\leq 55 \mathrm{mg}$ per deciliter $(3.1 \mathrm{mmol}$ per liter) than did the placebo group. However, the sotagliflozin group had a higher rate of diabetic ketoacidosis than that of the placebo group (3.0\% [21 patients] and $0.6 \%$ [4 patients], respectively). The overall rate of adverse events, regardless of cause, was similar between the two groups $(55.1 \%$ for sotagliflozin and $52.5 \%$ in the placebo group).

\section{Conclusions}

In this phase 3 trial in adults with T1D who were receiving insulin, the percentage of patients who achieved the primary endpoint of HbA1c level $<7.0 \%$ without any episodes of severe hypoglycemia or diabetic ketoacidosis was higher in the group that received sotagliflozin than it was in the placebo group. However, the rates of both hypoglycemia and diabetic ketoacidosis were higher in the group of patients receiving sotagliflozin.

\section{Effects of sotagliflozin added to insulin in type 1 diabetes}

Reply to comments on "Effects of sotagliflozin added to insulin in type 1 diabetes" [N Engl J Med. 2017]

Garg SK ${ }^{1}$, Strumph $P^{2}$

${ }^{1}$ University of Colorado Denver, Aurora, CO; ${ }^{2}$ Lexicon Pharmaceuticals, The Woodlands, TX

N Engl J Med 2018; 378: 966-968 
Table 1. Comparison of Rates of Diabetic Ketoacidosis in DEPICT-1 and inTandem3 Trials after Standardization of the Criteria Used

\begin{tabular}{|c|c|c|c|c|c|}
\hline \multirow[b]{2}{*}{ Variable } & \multicolumn{2}{|c|}{ inTandem $3^{1,2}$} & \multicolumn{3}{|c|}{$D E P I C T-1^{3}$} \\
\hline & $\begin{array}{c}\text { Placebo } \\
(N=703)\end{array}$ & $\begin{array}{l}\text { Sotagliflozin, } \\
400 \mathrm{mg} \\
(N=699)\end{array}$ & $\begin{array}{c}\text { Placebo } \\
(N=260)\end{array}$ & $\begin{array}{c}\text { Dapagliflozin, } \\
5 m g \\
(N=277)\end{array}$ & $\begin{array}{c}\text { Dapagliflozin, } \\
10 \mathrm{mg} \\
(N=296)\end{array}$ \\
\hline $\begin{array}{l}\text { All patients with } \\
\text { certain, definite, } \\
\text { probable, or } \\
\text { possible diabetic } \\
\text { ketoacidosis—no. }(\%)\end{array}$ & $4(0.6)$ & $21(3.0)$ & $4(1.5)$ & $9(3.2)$ & $12(4.1)$ \\
\hline $\begin{array}{l}\text { Percent difference } \\
\text { between treatment } \\
\text { and placebo among } \\
\text { patients with } \\
\geq 1 \text { event }\end{array}$ & - & 2.4 & - & 1.7 & 2.5 \\
\hline
\end{tabular}

DEPICT-1 denotes Dapagliflozin Evaluation in Patients with Inadequately Controlled Type 1 Diabetes.

${ }^{1}$ Garg SK, Strumph P. N Engl J Med. 2018; 378: 967-968.

${ }^{2}$ Garg SK, et al. N Engl J Med. 2017; 377: 2337-2348.

${ }^{3}$ Dandona P, et al. Lancet Diabetes Endocrinol. 2017; 5: 864-876.

Sotagliflozin in combination with optimized insulin therapy in adults with type 1 diabetes: the North American inTandem1 study

Buse $J B^{1}$, Garg $S K^{2}$, Rosenstock $J^{3}$, Bailey $T S^{4}$, Banks $P^{5}$, Bode $B W^{6}$, Danne $T^{7}$, Kushner $J A^{8}$, Lane $W S^{9}$, Lapuerta $\mathrm{P}^{5}$, McGuire $D K^{10}$, Peters $A L^{11}$, Reed $\mathrm{J}^{12}$, Sawhney $\mathrm{S}^{5}$, Strumph $P^{5}$

${ }^{I}$ Department of Medicine, University of North Carolina School of Medicine, Chapel Hill, NC; ${ }^{2}$ Departments of Medicine and Pediatrics, Barbara Davis Center for Diabetes, University of Colorado Denver, Aurora, CO; ${ }^{3}$ Dallas Diabetes Research Center at Medical City, Dallas, TX; ${ }^{4}$ AMCR Institute, Escondido, CA; ${ }^{5}$ Lexicon Pharmaceuticals, Inc., The Woodlands, TX; ${ }^{6}$ Atlanta Diabetes Associates, Emory University School of Medicine, Atlanta, GA; ${ }^{7}$ Department of Diabetes, Endocrinology, and Clinical Research, Children's and Youth Hospital Auf der Bult, Hannover Medical School, Hannover, Germany; ${ }^{8}$ McNair Medical Institute and Department of Pediatrics, Baylor College of Medicine and Texas Children's Hospital, Houston, TX; ${ }^{9}$ Mountain Diabetes and Endocrine Center, Asheville, NC; ${ }^{10}$ Department of Internal Medicine, University of Texas Southwestern Medical Center, Dallas, TX; ${ }^{11}$ Keck School of Medicine of the University of Southern California, Los Angeles, CA, ${ }^{12}$ Endocrine Research Solutions, Inc., Roswell, GA

Diabetes Care 2018; 41: 1970-1980

\section{Objective}

This trial investigated the safety and efficacy of the dual sodium-glucose cotransporter 1 (SGLT1) and SGLT2 inhibitor sotagliflozin when used in combination with optimized insulin in patients with T1D.

\section{Methods}

The inTandem 1 trial was a double-blind, 52-week phase 3 trial carried out in North American adult patients with T1D.
Following 6 weeks of insulin optimization, participants were randomly assigned to one of three treatments: placebo $(n=268)$, sotagliflozin $200 \mathrm{mg} \quad(n=263)$, or sotagliflozin $400 \mathrm{mg}(n=262)$. Change in HbA1c from baseline to 24 weeks was the primary endpoint, although $\mathrm{HbA1c}$ as well as weight and safety were assessed through 52 weeks.

\section{Results}

At 24 weeks, placebo-adjusted HbA1c decreased by $0.36 \%$ with sotagliflozin 200 and $0.41 \%$ with sotagliflozin $400 \mathrm{mg}$, and by $0.25 \%$ and $0.31 \%$, respectively, at 52 weeks (all $P<0.001)$. HbA1c $<7 \%$ was attained at 24 weeks by $15.7 \%$ of patients in the placebo group and $27.2 \%$ and $40.3 \%$ of those in the sotagliflozin $200 \mathrm{mg}$ and sotagliflozin $400 \mathrm{mg}$ groups, respectively $(P \leq 0.003$ vs placebo). Between sotagliflozin $400 \mathrm{mg}$ and placebo, mean treatment differences at 52 weeks were $-1.08 \mathrm{mmol} / \mathrm{L}$ for fasting plasma glucose, $-4.32 \mathrm{~kg}$ for weight, $-15.63 \%$ for bolus insulin dose, and $-11.87 \%$ for basal insulin dose (all $P<0.001$ ). Questionnaire scores for treatment satisfaction rose significantly $(+2.5$ points $)$ for sotagliflozin vs placebo $(P<0.001)$ at 24 weeks. Participants reported increased incidence of both genital mycotic infections and diarrhea with sotagliflozin. Adjudicated diabetic ketoacidosis (DKA) occurred in 3.4\% $(\mathrm{n}=9)$ and $4.2 \%$ $(\mathrm{n}=11)$ patients receiving sotagliflozin 200 and $400 \mathrm{mg}$, respectively, and in 1 patient $(0.4 \%)$ in the placebo group. Seventeen patients $(6.5 \%)$ from each sotagliflozin group (200 $\mathrm{mg}$ and $400 \mathrm{mg}$ dosage) experienced sever hypoglycemia, as did 26 patients $(9.7 \%)$ receiving placebo.

\section{Conclusions}

During this 1-year study in North American adults with T1D, the combination of sotagliflozin and optimized insulin therapy was correlated with sustained decrease in $\mathrm{HbA1c}$, weight reduction, lower insulin dose, a drop in the number of severe hypoglycemic episodes, and improvement in patientreported outcomes, but also more DKA, relative to placebo. 
HbA1c and hypoglycemia reductions at 24 and 52 weeks with sotagliflozin in combination with insulin in adults with type 1 diabetes: the European inTandem2 study

Danne $T^{l}$, Cariou $B^{2}$, Banks $P^{3}$, Brandle $\mathrm{M}^{4}$, Brath $\mathrm{H}^{5}$, Franek $E^{6}$, Kushner $J A^{7}$, Lapuerta $\mathrm{P}^{3}$, McGuire $D K^{8}$, Peters $A L^{9}$, Sawhney $S^{3}$, Strumph $\mathrm{P}^{3}$

${ }^{I}$ Department of Diabetes, Endocrinology, and Clinical Research, Children's and Youth Hospital Auf der Bult, Hannover Medical School, Hannover, Germany; ${ }^{2}$ l'Institut du thorax, Department of Endocrinology, INSERM, Nantes, France; ${ }^{3}$ Lexicon Pharmaceuticals, Inc., The Woodlands, TX; ${ }^{4}$ Department of Internal Medicine, Kantonsspital St. Gallen, St. Gallen, Switzerland; ${ }^{5}$ Diabetes Outpatient Clinic, Health Center South, Vienna, Austria; ${ }^{6}$ Mossakowski Medical Research Center, Polish Academy of Sciences, Warsaw, Poland; ${ }^{7}$ Department of Pediatrics, Baylor College of Medicine and Texas Children's Hospital, Houston, TX; ${ }^{8}$ Department of Internal Medicine, Division of Cardiology, University of Texas Southwestern Medical Center, Dallas, TX; ${ }^{9}$ University of Southern California Keck School of Medicine, Los Angeles, CA

Diabetes Care 2018; 41: 1981-1990

\section{Objective}

The efficacy and safety of the dual SGLT1 and SGLT2 inhibitor sotagliflozin vs placebo when combined with optimized insulin in adults with T1D was investigated.

\section{Research Design and Methods}

A 52-week, randomized, double-blind, international phase 3 trial was conducted in adults with T1D. After 6 weeks of insulin optimization, subjects were randomly assigned to one of three treatment groups: placebo $(n=258)$, once-daily oral sotagliflozin $200 \mathrm{mg}(n=261)$, or once-daily oral sotagliflozin $400 \mathrm{mg}(n=263)$. Change in HbAlc from baseline to 24 weeks was the primary endpoint. The first secondary endpoint, at week 24 , was a composite of the proportion of patients with $\mathrm{HbA} 1 \mathrm{c}<7.0 \%$ and no episodes of either severe hypoglycemia or ketoacidosis (DKA). Additional endpoints (fasting glucose, weight, insulin dose, and safety) were evaluated through 52 weeks.

\section{Results}

Placebo-adjusted changes in $\mathrm{HbA1c}$ from baseline at 24 weeks $(7.8 \%)$ were $-0.37 \%$ with sotagliflozin $200 \mathrm{mg}$ and $-0.35 \%$ with sotagliflozin $400 \mathrm{mg}(P<0.001)$, and differences were maintained at 52 weeks. At 52 weeks, compared with placebo, larger percentages of patients receiving sotagliflozin met the composite endpoint $(25.67 \%$ and $26.62 \%$ for sotagliflozin $22 \mathrm{mg}$ and $400 \mathrm{mg}$, respectively, vs $14.34 \%$ for placebo; $P \leq 0.001$ ). Additionally, sotagliflozin $400 \mathrm{mg}$ reduced fasting plasma glucose $(-0.87 \mathrm{mmol} / \mathrm{L} ; P=0.008)$, weight $(-2.92 \mathrm{~kg} ; P<0.001)$, and total daily insulin dose $(-8.2 \% ; P=0.001)$. A 24-week substudy of continuous glucose monitoring (CGM) found a decrease in postprandial glucose $(P \leq 0.009)$ as well as more time (up to $3 \mathrm{~h})$ in the target range of 3.9-10.0 mmol/L with sotagliflozin. Participants reported increased treatment satisfaction and reduced diabetes distress with sotagliflozin $(P<0.05$ vs placebo), as well as a lower frequency of documented hypoglycemia. In sotagliflozin treatment groups, severe hypoglycemia occurred by week 52 in 13 patients $(5.0 \%)$ at $200 \mathrm{mg}$ and 13 patients $(5.0 \%)$ at $400 \mathrm{mg}$, while severe hypoglycemia occurred in 6 patients $(2.3 \%)$ treated with placebo. DKA occurred in 0 of 258 patients treated with sotagliflozin at $200 \mathrm{mg}, 6$ of 261 patients $(2.3 \%)$ at $400 \mathrm{mg}$, and 9 of 263 patients (3.4\%) treated with placebo.

\section{Conclusions}

During this 1-year study, sotagliflozin was associated with statistically significant decreases in HbAlc. Patients using sotagliflozin experienced more episodes of DKA but fewer episodes of documented and severe hypoglycemia relative to those receiving placebo.

\section{Efficacy and safety of dapagliflozin in patients with inadequately controlled type 1 diabetes (DEPICT-1): 24 week results from a multicentre, double-blind, phase 3 , randomised controlled trial}

Dandona $P^{1}$, Mathieu $C^{2}$, Phillip $M^{3}$, Hansen $L^{4}$, Griffen $S C^{5}$, Tschöpe $D^{6}$, Thorén $F^{7}, X u J^{8}$, Langkilde $A M^{7}$; DEPICT-1 Investigators

${ }^{I}$ Department of Medicine, State University of New York at Buffalo, Buffalo, NY; ${ }^{2}$ Clinical and Experimental Endocrinology, UZ Gasthuisberg, University of Leuven, Leuven, Belgium; ${ }^{3}$ Schneider Children's Medical Center of Israel, Institute for Endocrinology and Diabetes, Petah Tikva, Israel; Sackler Faculty of Medicine, Tel-Aviv University, Tel-Aviv, Israel; ${ }^{4}$ Bristol-Myers Squibb, Princeton, NJ; ${ }^{5} J D R F$, New York, NY; ${ }^{6}$ Department for Endocrinology, Diabetology and Gastroenterology, Heart and Diabetes Centre, Bad Oeynhausen, Germany; Ruhr University Bochum, Bochum, Germany; ${ }^{7}$ AstraZeneca, Mölndal, Sweden; ${ }^{8}$ AstraZeneca, Gaithersburg, MD

Lancet Diabetes Endocrinol 2017; 5: 864-876

\section{Background}

Dapagliflozin is a sodium-glucose cotransporter 2 (SGLT2) inhibitor approved for the treatment of T2D. This trial in adults with inadequately controlled T1D evaluated the efficacy and safety of dapagliflozin as an add-on to adjustable insulin.

\section{Methods}

DEPICT-1 was a double-blind, randomized, parallelcontrolled, three-arm, phase 3 multicenter study conducted at 143 sites in 17 countries. To be eligible, patients had to be 1875 years of age, with insufficiently controlled T1D (HbA1c between $\geq 7.7 \%$ and $\leq 11.0 \%$ [ $\geq 61.0 \mathrm{mmol} / \mathrm{mol}$ and $\leq 97.0$ $\mathrm{mmol} / \mathrm{mol}]$ ), who had been prescribed insulin for at least 12 months prior to enrollment. Diabetes management was optimized during an 8-week lead-in period. After the optimization period, an interactive voice response system was used to randomize participants $(1: 1: 1)$ to oral dapagliflozin once daily at a dose $5 \mathrm{mg}$ or $10 \mathrm{mg}$, or to a matched placebo. Current use of CGM, method of insulin administration, and baseline HbA1c were used to stratify randomization. The primary efficacy endpoint was the change in HbAlc from baseline after 24 weeks of treatment in the full analysis set, 
which comprised all randomly assigned patients who were administered at least one dose of study drug. The safety analysis set included 55 additional patients who were incorrectly and nonrandomly allocated to only dapagliflozin treatment groups. Data collection was completed on January 4,2017 , for the current investigation, and a 28 -week extension phase is ongoing.

\section{Findings}

Between November 11, 2014, and April 16, 2016, a total of 833 patients were assigned to treatment groups and included in safety analyses (dapagliflozin $5 \mathrm{mg}[\mathrm{n}=277]$ vs dapagliflozin $10 \mathrm{mg}[\mathrm{n}=296]$ vs placebo $[\mathrm{n}=260] ; 778$ of these patients were randomly assigned and included in the full analysis set for efficacy analyses (259 vs 259 vs 260), and 55 were only included in the safety analysis due to a randomization error. Mean baseline HbA1c was $8.53 \%$ (70 mmol/mol; SD $0.67 \%$ [7.3 $\mathrm{mmol} / \mathrm{mol}]$ ). HbA1c was significantly reduced by both doses of dapagliflozin compared with placebo at week 24: mean baseline to week 24 difference for dapagliflozin $5 \mathrm{mg}$ vs placebo, $-0.42 \%$ ([95\% CI -0.56 to -0.28$] ; P<0.0001)$, and for dapagliflozin $10 \mathrm{mg}$ vs placebo, $-0.45 \%$ ([95\% CI -0.58 to -0.31 ] $; P<0.0001)$. Several adverse effects were measured among all subjects in the dapagliflozin $5 \mathrm{mg}(\mathrm{n}=277)$, dapagliflozin $10 \mathrm{mg}(\mathrm{n}=296)$, and placebo $(\mathrm{n}=260)$ groups. The most commonly reported adverse events were nasopharyngitis (38 [14\%] vs 36 [12\%] vs 39 [15\%], respectively); urinary tract infection (19 [7\%] vs 11 [4\%] vs 13 [5\%]); upper respiratory tract infection (15 [5\%] vs $15[5 \%]$ vs $11[4 \%])$, and headache (12 [4\%] vs 17 [6\%] vs 11 [4\%]). Hypoglycemia was recorded in 220 (79\%), 235 (79\%), and $207(80 \%)$ subjects in the dapagliflozin $5 \mathrm{mg}$, dapagliflozin $10 \mathrm{mg}$, and placebo groups, respectively. Severe hypoglycemia was reported in $21(8 \%)$, $19(6 \%)$, and $19(7 \%)$ subjects, respectively. Adjudicated definite DKA occurred in $4(1 \%)$ participants in the dapagliflozin $5 \mathrm{mg}$ group, $5(2 \%)$ in the dapagliflozin $10 \mathrm{mg}$ group, and $3(1 \%)$ in the placebo group.

\section{Conclusion}

The results of this phase 3 trial indicate that the use of dapagliflozin as an adjunct treatment can improve glycemic control in patients with inadequately controlled T1D who are already using insulin.

\section{Cardiovascular mortality and morbidity in patients with type 2 diabetes following initiation of sodium- glucose co-transporter-2 inhibitors versus other glucose-lowering drugs (CVD-REAL Nordic): a multinational observational analysis}

Birkeland $\mathrm{KI}^{1}$, Jørgensen $\mathrm{ME}^{2}$, Carstensen $\mathrm{B}^{3}$, Persson $F^{3}$, Gulseth $\mathrm{HL}^{4}$, Thuresson $\mathrm{M}^{5}$, Fenici $P^{6}$, Nathanson $D^{7}$, Nyström $T^{7}$, Eriksson $\mathrm{JW}^{8}$, Bodegård $J^{9}$, Norhammar $A^{10}$

${ }^{1}$ Institute of Clinical Medicine, University of Oslo, Oslo, Norway; Department of Transplantation Medicine, Oslo University Hospital, Oslo, Norway; ${ }^{2}$ Steno Diabetes Center Copenhagen, Gentofte, Denmark; National Institute of Public Health, Southern Denmark University, Copenhagen, Denmark; ${ }^{3}$ Steno Diabetes Center Copenhagen, Gentofte, Denmark; ${ }^{4}$ Department of Endocrinology, Morbid Obesity and Preventive Med- icine, Oslo University Hospital, Oslo, Norway; ${ }^{5}$ Statisticon AB, Uppsala, Sweden; ${ }^{6}$ AstraZeneca, Cambridge, UK; ${ }^{7}$ Department of Clinical Science and Education, Karolinska Institutet, Stockholm, Sweden; ${ }^{8}$ Department of Medical Sciences, Uppsala University, Uppsala, Sweden; ${ }^{9}$ Medical Department, AstraZeneca Nordic-Baltic, Oslo, Norway; ${ }^{10}$ Department of Medicine, Södersjukhuset, Karolinska Institutet, Stockholm, Sweden; Capio St Görans Hospital, Stockholm, Sweden

Lancet Diabetes Endocrinol 2017; 5: 709-717

\section{Background}

The use of SGLT2 inhibitors empagliflozin and canagliflozin in treating patients with T2D and a high cardiovascular risk profile has been shown to decrease cardiovascular morbidity and mortality. This study uses real-world data from clinical practice in patients with a broad cardiovascular risk profile to compare cardiovascular mortality and morbidity in new users of SGLT2 inhibitors and new users of other glucose-lowering drugs.

\section{Methods}

CVD-REAL Nordic was an observational analysis of individual patient-level data from the prescribed drug, cause of death, and national patient registries in Denmark, Norway, and Sweden. Any patients filling a prescription for glucoselowering drugs between 2012 and 2015 were entered in the study and followed up until December 31, 2015. Subjects were separated into two categories: new users of SGLT2 inhibitors and new users of other glucose-lowering drugs. Propensity scores were used to match each user of SGLT2 inhibitor with three users of other drugs for lowering glucose. Hazard ratios (HRs) were estimated by country (Cox survival model) and weighted averages were calculated. Cardiovascular mortality, major adverse cardiovascular events (cardiovascular mortality, myocardial infarction, and ischemic or hemorrhagic stroke), hospital events for heart failure (inpatient or outpatient visit with a primary diagnosis of heart failure), nonfatal myocardial infarction, nonfatal stroke, and atrial fibrillation were examined. Incidence of severe hypoglycemia was also evaluated.

\section{Results}

Matched SGLT2 inhibitor $(n=22,830)$ and other glucoselowering drug $(n=68,490)$ groups were well balanced at baseline, with a mean follow-up of 0.9 (SD 4.1) years $(80,669$ patient-years) and mean age of 61 (SD 12.0) years. Women made up $40 \%(36,362 / 91,320)$ of the study population, and the frequency of cardiovascular disease was $25 \%(22,686 /$ 91,320). Of the overall exposure time for any SGLT2 inhibitor dapagliflozin accounted for $94 \%$, with $5 \%$ for empagliflozin and $1 \%$ for canagliflozin. SGLT2 inhibitors were associated with reduced risk of cardiovascular mortality compared with other glucose-lowering drugs (HR 0.53 [95\% CI 0.40-0.71]), major adverse cardiovascular events $(0.78$ [0.69-0.87]), and hospital events for heart failure $(0.70$ [0.61$0.81] ; P<0.0001$ for all). No significant differences were found between treatment with SGLT2 inhibitors and other glucose-lowering drugs with respect to nonfatal myocardial infarction or stroke or atrial fibrillation. SGLT2 inhibitors correlated with a reduced risk of severe hypoglycemia vs other glucose-lowering drugs (HR 0.76 [0.65-0.90]; $P=0.001)$. 
Differences in cardiovascular mortality were similar for subjects with cardiovascular disease (25\% of total) at baseline and those without (HR 0.60 [0.42-0.85] vs 0.55 [0.34-0.90]). HR for major adverse cardiovascular events in the group who had cardiovascular disease at baseline was $0.70(0.59-0.83)$ vs 0.90 (0.76-1.07) in the group without.

\section{Conclusions}

The findings for this population of patients were consistent with the results of clinical trials in patients with high cardiovascular risk. Subjects with T2D and a broad risk profile for cardiovascular events, and use of an SGLT2 inhibitor were associated with decreased cardiovascular disease and cardiovascular mortality compared with use of other glucose lowering.

\section{Canagliflozin in conjunction with sulfonylurea maintains glycemic control and weight loss over 52 weeks: a randomized, controlled trial in patients with type 2 diabetes mellitus}

Yale $J F^{1}$, Xie $J^{2}$, Sherman $S E^{3}$, Garceau $C^{4}$

${ }^{I}$ Department of Medicine, McGill University, Montreal, Quebec, Canada; ${ }^{2}$ Janssen Research and Development, Raritan, NJ; ${ }^{3}$ Janssen Inc., Toronto, Ontario, Canada; ${ }^{4}$ Institut Universitaire de Cardiologie et de Pneumologie de Québec, Quebec City, Quebec, Canada

Clin Ther 2017; 39: 2230-2242

\section{Background}

This trial examined the long-term efficacy and safety of canagliflozin, an inhibitor of SGLT2, added to background monotherapy using sulfonylurea (SU) for patients with type 2 diabetes mellitus.

\section{Methods}

The CANagliflozin cardiovascular Assessment Study (CANVAS) was a randomized, double-blind, placebocontrolled cardiovascular outcomes study that assigned participants at random to have one daily dose of placebo, canagliflozin $100 \mathrm{mg}$, or canagliflozin $300 \mathrm{mg}$ added to their routine therapy. Included in CANVAS was a prespecified SU substudy of subjects taking background doses of SU monotherapy, and data from the primary assessment of efficacy at 18 weeks have been reported previously. A retrospective analysis of the SU substudy at 52 weeks evaluated long-term efficacy and safety of canagliflozin used with an SU, with a primary objective of assessing the change in $\mathrm{HbA1c}$ from baseline to 52 weeks.

\section{Results}

The 52-week extension study included 215 patients in total. Patients who were given either 100-mg or 300-mg doses of canagliflozin experienced a sustained reduction in $\mathrm{HbA1c}$ vs placebo $(-0.61 \%$ [95\% CI $-0.941 \%$ to $-0.282 \%$ ] for $100 \mathrm{mg}$ and $-0.66 \%$ [95\% CI, $-0.993 \%$ to $-0.332 \%$ ] for $300 \mathrm{mg}$ ), irrespective of baseline $\mathrm{HbA1c}$, SU dose, duration of diabetes, estimated glomerular filtration rate, or body mass index. Both canagliflozin groups (100-mg and 300-mg dosages) experienced sustained reduction in fasting plasma glucose relative to placebo
$(-2.04 \mathrm{mmol} / \mathrm{L}$ [95\% CI -2.778 to $-1.299 \mathrm{mmol} / \mathrm{L}]$ for $100 \mathrm{mg}$ and $-1.88 \mathrm{mmol} / \mathrm{L}$ [95\% CI, -2.623 to $-1.146 \mathrm{mmol} /$ $\mathrm{L}$ ] for $300 \mathrm{mg}$ ). Weight was significantly reduced at 52 weeks in both 100-mg and 300-mg groups compared with placebo (weight reduction $-1.9 \%$ [95\% CI $-3.2 \%$ to $-0.7 \%]$ and $-2.0 \%$ [95\% CI $-3.2 \%$ to $-0.7 \%$ ], respectively). Lowered systolic blood pressure was also reported for both doses of canagliflozin relative to placebo; however, no clear difference was found in levels of high-density or low-density lipoprotein cholesterol or triglycerides. Overall, canagliflozin was well tolerated. Hypoglycemic events were documented in $14 \%$ of patients on placebo, and a similar frequency was found in patients who received canagliflozin. In both canagliflozin groups combined, there was an increased frequency of genital mycotic infections in both men $(5.1 \%)$ and women $(10.4 \%)$ relative to the placebo group $(0 \%)$, with a greater increase in the higher-dose group. A slightly higher rate of renal impairment was measured among those treated with canagliflozin vs placebo $(2.1 \%$ vs $0 \%)$.

\section{Implications}

After 52 weeks, patients receiving canagliflozin in addition to background SU had sustained decreases in both $\mathrm{HbA1c}$ and fasting plasma glucose, without an increase in hypoglycemia or body weight. Additionally, results of the safety evaluation were generally consistent with the known safety profile of canagliflozin.

\section{Dapagliflozin/saxagliptin fixed-dose tablets: a new sodium-glucose cotransporter 2 and dipeptidyl peptidase 4 combination for the treatment of type 2 diabetes}

Coppenrath $V A^{1}$, Hydery $T^{2}$

${ }^{1}$ Massachusetts College of Pharmacy and Health Sciences (MCPHS) University, Worcester, MA; ${ }^{2}$ UMass Medical School-Clinical Pharmacy Services, Shrewsbury, MA

Ann Pharmacother 2018; 52: 78-85

\section{Background}

The pharmacology, pharmacokinetics, efficacy, safety, and place in therapy of the fixed-dose combination (FDC) product QTERN (dapagliflozin/saxagliptin) tablets were investigated.

\section{Methods}

Searches of the MEDLINE online database (articles published from 1946 to July 1, 2017) were executed using three keywords: QTERN, saxagliptin, and dapagliflozin, and all English-language articles reporting on pharmacology, pharmacokinetics, efficacy, or safety of the combination therapy in human subjects were reviewed. Additional data were obtained from Clinicaltrials.gov as well as the QTERN prescribing information and product dossier.

\section{Results}

There was no significant effect on the pharmacokinetics of saxagliptin and dapagliflozin when the two drugs were administered as an FDC product. Saxagliptin may act to inhibit the dapagliflozin-associated increase in glucagon secretion. 
Studies have been carried out on the dapagliflozin/saxagliptin combination as an add-on to metformin treatment in patients with uncontrolled type 2 diabetes mellitus (T2DM). The difference in HbA1c between saxagliptin + dapagliflozin + metformin (triple therapy) and saxagliptin + metformin was -0.59 ([95\% CI -0.81 to -0.37$] P<0.0001)$; between triple therapy and dapagliflozin + metformin, the difference was -0.27 ( $[95 \%$ CI -0.48 to -0.05$], P=0.0166)$. The FDC QTERN was well tolerated when used as an add-on therapy with metformin.

\section{Conclusions}

QTERN (dapagliflozin/saxagliptin) tablets are a valid option for patients with T2DM not controlled with metformin alone; however, cost, insurance coverage, and a mediocre reduction in HbA1c will likely limit its use until there are more data available with respect to its effects on diabetes complications and cardiovascular outcomes.

\section{Sodium glucose co-transporter 2 inhibitor luseogliflozin in the management of type 2 diabetes: a drug safety evaluation}

Yabe $D^{1}$, Hamamoto $Y^{1,2,3}$, Seino $Y^{4}$, Kuwata $H^{1,2}$, Kurose $T^{1,2}$, Seino $Y^{1,2}$

${ }^{1}$ Yutaka Seino Distinguished Center for Diabetes Research, Kansai Electric Power Medical Research Institute, Kobe, Japan; ${ }^{2}$ Center for Diabetes, Endocrinology and Metabolism, Kansai Electric Power Hospital, Osaka, Japan; ${ }^{3}$ Center for Metabolism and Clinical Nutrition, Kansai Electric Power Hospital, Osaka, Japan; ${ }^{4}$ Departments of Endocrinology and Diabetes, Nagoya University Graduate School of Medicine, Nagoya, Japan

Expert Opin Drug Saf 2017; 16: 1211-1218

\section{Introduction}

Recently developed sodium glucose co-transporter-2 (SGLT2) inhibitors present a new class of antidiabetic drug that is increasingly employed in the management of T2D. The glucose-lowering mechanism of SGLT2 inhibitors differs significantly from the mechanisms used by other antidiabetic drugs; therefore, safe use of these new drugs needs to be examined based on results from both preapproval clinical trials and real-world studies. The SGLT2 inhibitor luseogliflozin was developed by Taisho Pharmaceutical Co., Ltd. and was approved as an oral antidiabetic drug for T2D in Japan.

\section{Areas covered}

A literature review was conducted to collect data regarding the overall safety and efficacy of the SGLT2 inhibitor luseogliflozin, the results of which are summarized, with a focus on adverse drug reactions reported in preapproval clinical trials and post-marketing surveillance.

\section{Expert opinion}

In preapproval clinical trials, luseogliflozin was shown to be well tolerated and significantly improved hyperglycemia. Additionally, the safety profile of this SGLT2 inhibitor in elderly patients was found to be favorable in both preapproval clinical trials and post-marketing surveillance. While longterm safety and efficacy are yet to be discovered, luseogliflozin can benefit T2D patients worldwide. To ensure patient safety and effective results, health-care professionals must perform appropriate patient education and temporarily withdraw luseogliflozin during patient "sick day" as well as avoiding strict carbohydrate restriction during luseogliflozin treatment.

\section{Effects of sodium glucose cotransporter-2 inhibitors on serum uric acid in type 2 diabetes mellitus}

Ahmadieh $H^{1}$, Azar $S^{2}$

${ }^{1}$ Clinical Sciences Department, Internal Medicine Department, Faculty of Medicine, Beirut Arab University, Beirut, Lebanon; ${ }^{2}$ Division of Endocrinology, Department of Internal Medicine, American University of Beirut, New York, NY

Diabetes Technol Ther 2017; 19: 507-512

\section{Background}

Hyperuricemia has been linked to metabolic syndrome, cardiovascular disease, and chronic kidney disease. Hyperuricemia and type 2 diabetes mellitus (T2DM) were interrelated, T2DM patients were more at risk of having a higher serum uric acid level, and individuals with higher serum uric acid had higher risk of developing T2D in the future. Insulin resistance seems to play an important role in the causal relationship between metabolic syndrome, T2D, and hyperuricemia. Oral diabetic drugs that would have additional beneficial effects in the reduction of serum uric acid levels are of importance.

\section{Results}

Selective SGLT2 inhibitors were extensively studied in T2DM and were found to offer improvement of glycemic control in addition to their proven metabolic effects on weight and blood pressure. The additional beneficial effect of SGLT2 inhibitors on serum uric acid level reduction is also investigated. Recently, data have been accumulating showing that SGLT2 inhibitors have additional beneficial effects on serum uric acid reduction.

\section{Conclusions}

As for the postulated mechanism, serum uric acid decreased in patients using SGLT2 inhibitor as a result of the increased urinary excretion rate of uric acid, owing to the inhibition of uric acid reabsorption mediated by the effect of the drug on the GLUT9 isoform 2, located at the collecting duct of the renal tubule.

\section{Altered patterns of early metabolic decompensation in type 1 diabetes during treatment with a SGLT2 inhibitor: an insulin pump suspension study}

Patel NS, Van Name MA, Cengiz E, Carria LR, Weinzimer SA, Tamborlane WV, Sherr JL

Department of Pediatric Endocrinology, Yale School of Medicine, New Haven, CT

Diabetes Technol Ther 2017; 19: 618-622 


\section{Background}

Enthusiasm for the benefits of sodium-glucose cotransporter 2 (SGLT2) inhibitors as an adjunctive treatment in T1D has been offset by the possible increased risk of diabetic ketoacidosis (DKA). Since pump-treated T1D patients are susceptible to DKA due to infusion site problems, this study was undertaken to assess how treatment with SGLT2 inhibitors affects patterns of early metabolic decompensation following suspension of basal insulin.

\section{Methods}

Ten T1D participants (age 19-35 years, duration 10 18 years, HbA1c $7.4 \% \pm 0.8 \%$ ) underwent overnight pump suspension studies before and after treatment with canagliflozin (CANA). On both nights, basal insulin was suspended at 3 a.m. and plasma glucose (PG), $\beta$-hydroxybutyrate $(\mathrm{BHB})$, free fatty acids (FFA), plasma insulin, and glucagon were measured. Studies were terminated 6 hours after suspension or if $\mathrm{PG}$ rose to $>350 \mathrm{mg} / \mathrm{dL}$ or $\mathrm{BHB}$ to $>2.5 \mathrm{mmol} / \mathrm{L}$.

\section{Results}

Plasma insulin levels at the start of suspension were reduced by $30 \%$ after CANA treatment $(44 \pm 11 \mu \mathrm{U} / \mathrm{mL}$ vs $31 \pm 10 \mu \mathrm{U} / \mathrm{mL}, P<0.01)$, but baseline PG, BHB, FFA, and glucagon levels were not significantly different. During the suspension, PG rose from $104 \pm 10$ to $301 \pm 21 \mathrm{mg} / \mathrm{dL}$ before treatment, but only from $109 \pm 8$ to $195 \pm 14 \mathrm{mg} / \mathrm{dL}$ after treatment ( $P=0.002$ vs pretreatment values). On the other hand, CANA treatment did not significantly affect the magnitude of increases in FFA, BHB, and glucagon levels during the suspension study.

\section{Conclusion}

These data indicate that SGLT2 inhibitors do not accelerate the rate of ketogenesis following the interruption of basal insulin infusion in T1D. Rather, the failure of patients to promptly recognize early metabolic decompensation relates to the much more gradual rise in PG levels.

\section{Comments}

A majority of the patients with type 1 diabetes are getting overweight/obese (67\% T1D Exchange follow-up data), and nearly $70 \%$ of patients do not achieve target HbAlc levels with insulin treatment alone. The T1D Exchange 2018 data also show that only a small number of patients $(<2 \%)$ are using adjunctive therapies (off-label) for T1D management. The only approved adjunctive therapy for T1D is pramlintide, which is rarely prescribed due to gastrointestinal (GI) side effects and the risk of severe hypoglycemia. Most of the adjunctive therapies in randomized control trials (metformin, GLP-1 analogs, coleselvelam, and dipeptidyl peptidase-IV [DPP-IV] inhibitors) for the treatment of patients with T1D have not proven to be successful in achieving improved glycemic control and/or weight loss without increasing hypoglycemia.

Several studies including SGLT1/2 and SGLT2 inhibitors showed significant efficacy in adjunctive treatment of patients with T1D. Studies on sotagliflozin (InTandem 1, 2, and 3), which is an SGLT1/2 inhibitor, showed an increased number of patients (nearly 2-fold) reaching target $\mathrm{HbA} 1 \mathrm{cs}(<7 \%)$ without any increase in severe hypoglycemia or diabetic ketoacidosis ("net clinical benefit"). In addition, patients with T1D lost weight in a 52-week double-blind, randomized, controlled trial using sotagliflozin, with weight loss approaching about 3\%-4\% from baseline body weight. Similar studies done with dapagliflozin (DEPICT 1 and 2) also showed a significant decline in HbA1c and weight loss with no increase in hypoglycemia. The studies on dapagliflozin were slightly different than the studies using sotagliflozin, such that patients achieving HbA1c levels below $7.5 \%$ at the end of the optimization phase were excluded from being randomized in the dapagliflozin studies, whereas in the InTandem 1 and 2 using sotagliflozin, patients were randomized in the study irrespective of their drop in HbA1c levels after 6 weeks of optimization. The studies using both compounds showed a significant reduction in total insulin dose, with a larger reduction in basal insulin dose with dapagliflozin vs increased reductions in bolus insulin dose with sotagliflozin. The differences in the insulin dose reductions might be relevant due to SGLT1 effect in sotagliflozin on the upper-GI tract, where there is a decreased and/or delayed glucose absorption from the upper intestine. Since more glucose is presented to the latter part of the GI tract, it increases GLP-1 and peptide YY (also known as peptide tyrosine tyrosine) levels. Studies using both compounds showed a significant increase in time-in-range using CGM data.

SGLT2 inhibitors have a warning by the FDA in terms of increased risk of DKA in patients with T2D. As expected, both compounds showed a high risk of DKA in patients with T1D. The overall increase in risk of DKA was $2 \%-4 \%$, which is similar to the risk reported for the general population with T1D. However, there is no doubt that in these double-blind, randomized, controlled trials there was a definite increase in DKA compared with the placebos. Both of these drugs are with the U.S. FDA and European Medicines Agency for possible approval as adjunctive therapy for T1D. Another SGLT2 inhibitor (not approved in the United States or Western Europe) has also been submitted for approval for patients with T1D in Japan.

If regulatory agencies give approval for these drugs to be used adjunctively for patients with T1D with an aim to improve their glucose outcomes, it is imperative that DKA mitigation plans need to be in place to reduce the overall risk of DKA.

The real-world studies using empagliflozin and canagliflozin documented reduced cardiovascular mortality as compared with other glucose-lowering drugs (CVDREAL Nordic study). Studies using FDCs of dapagliflozin and DPP-IV inhibitor (saxagliptin) demonstrated better HbA1c reductions and cardiovascular safety; however, the cost might be a limiting factor in the use of this combination. SGLT2 inhibitors have also been shown to have lower serum uric acid levels due to inhibition of uric acid reabsorption from the renal tubules. 


\section{Efficacy and Safety of Biosimilar SAR342434 insulin lispro in adults with type 1 diabetes also using insulin glargine-SORELLA 1 study}

Garg $S K^{1}$, Wernicke-Panten $K^{2}$, Rojeski $M^{3}$, Pierre $S^{4}$, Kirchhein $Y^{2}$, Jedynasty $K^{5}$

${ }^{1}$ Barbara Davis Center for Diabetes, University of Colorado Denver, Aurora, CO; ${ }^{2}$ Sanofi-Aventis Deutschland GmbH, Frankfurt, Germany; ${ }^{3}$ Sanofi, Bridgewater, NJ; ${ }^{4}$ Sanofi, Paris, France; ${ }^{5}$ Centrum Diabetologiczne, Centralny Szpital Kliniczny MSW, Warsaw, Poland

Diabetes Technol Ther 2017; 19: 516-526

\section{Background}

SAR342434 is a biosimilar (follow-on form) of insulin lispro-Humalog. This study aimed to show similar efficacy, safety, and immunogenicity of SAR342434 (SAR-Lis) vs insulin lispro-Humalog (Ly-Lis) in adult patients with T1D treated with multiple daily injections while using basal insulin glargine (Lantus; Gla-100).

\section{Methods}

SORELLA-1 was a randomized, open-label, phase 3 study (NCT02273180). Patients completing the 6-month main study continued on SAR-Lis or Ly-Lis, as randomized, for a 6-month safety extension. Assessments included change in HbAlc, fasting plasma glucose, seven-point self-monitored plasma glucose (SMPG) profiles, hypoglycemic events, treatmentemergent adverse events, and anti-insulin antibodies (AIAs).

\section{Results}

Five hundred seven patients were randomized (SAR-Lis $\mathrm{n}=253$; Ly-Lis $\mathrm{n}=254$ ). Least square (LS) mean (SEM) change in glycosylated hemoglobin (HbA1c) (baseline to week 26; primary endpoint) was similar in both treatment groups (SAR-Lis $-0.42 \%$, SEM 0.051; Ly-Lis, $-0.47 \%$ SEM 0.050). Noninferiority at the prespecified $0.3 \%$ noninferiority margin and inverse noninferiority were demonstrated (LS mean difference of SAR-Lis vs Ly-Lis $0.06 \%$ [ $95 \%$ confidence interval -0.084 to 0.197 ]). At week 52 (end of extension period) vs week 26, a small HbA1c increase was observed in both groups. Fasting plasma glucose and seven-point self-monitored plasma glucose profile changes, including postprandial glucose excursions, were similar between groups. At week 52, similar changes in mean daily mealtime and basal insulin doses were observed. Hypoglycemia, treatment-emergent adverse events, and AIAs (incidence, prevalence) did not differ between groups.

\section{Conclusions}

Results from this controlled study in patients with T1D also using Gla-100 support similar efficacy and long-term safety (including immunogenicity) of SAR-Lis and Ly-Lis.

\section{Efficacy and safety of MK-1293 insulin glargine compared with originator insulin glargine (Lantus) in type 1 diabetes: a randomized, open-label clinical trial}

Home $P D^{1}$, Lam $R L H^{2}$, Carofano $W L^{2}$, Golm $G T^{2}$, Eldor $R^{2}$, Crutchlow $M F^{2}$, Marcos $M C^{2}$, Rosenstock $\mathrm{J}^{3}$, Hollander $P A^{4}$, Gallwitz $B^{5}$
${ }^{I}$ Newcastle University, Newcastle upon Tyne, UK; ${ }^{2}$ Merck and Co., Inc., Kenilworth, NJ; ${ }^{3}$ Dallas Diabetes Research Center at Medical City, Dallas, TX; ${ }^{4}$ Baylor Endocrine Center, Dallas, TX; ${ }^{5}$ University of Tübingen, Tübingen, Germany

Diabetes Obes Metab 2018; 20: 2220-2228

\section{Background}

The efficacy and safety of biosimilar MK-1293 insulin glargine (Mk-Gla; $100 \mathrm{U} / \mathrm{mL}$ ) with originator insulin glargine, Lantus (Sa-Gla), are compared in patients with type 1 diabetes mellitus (T1DM).

\section{Methods}

A 52-week phase 3, randomized, active-controlled, openlabel study (clinicaltrials.gov NCT02059161) was conducted in 508 patients with T1DM (HbA1c $\leq 11.0 \%$; $97 \mathrm{mmol} / \mathrm{mol}$ ) taking basal and prandial insulin. Participants were randomly assigned $(1: 1)$ to once-daily Mk-Gla $(n=245)$ or Sa-Gla $(n=263)$. A pre-breakfast plasma glucose dosing algorithm was used to titrate the basal insulin dosage. Assessment of the noninferiority of $\mathrm{HbA} 1 \mathrm{c}$ change from baseline (margin of $0.40 \%$ [ $4.4 \mathrm{mmol} / \mathrm{mol}]$ ) for Mk-Gla vs Sa-Gla over 24 weeks. The primary safety objective was assessment of AIA development over 24 weeks.

\section{Results}

At week 24, the LS mean change from baseline for HbA1c was $-0.62[95 \% \mathrm{CI}-0.79,-0.45] \%(-6.8[-8.7,-4.9]$ $\mathrm{mmol} / \mathrm{mol})$ for Mk-Gla and $-0.66[-0.82,-0.50] \%(-7.2$ $[-9.0,-5.4] \mathrm{mmol} / \mathrm{mol})$ for Sa-Gla. The LS mean HbA1c difference for Mk-Gla minus Sa-Gla was 0.04 [-0.11, $0.19] \%(0.4[-1.2,2.0] \mathrm{mmol} / \mathrm{mol})$, which meets the primary and secondary objectives' noninferiority and equivalence criteria. The mean dosages of insulin glargine for Mk-Gla and Sa-Gla were 0.46 and $0.48 \mathrm{U} / \mathrm{kg}$, respectively. The similarity between $\mathrm{HbA} 1 \mathrm{c}$ response and basal insulin dose trajectories continued over the full 52 weeks. Over the duration of the study, Mk-Gla and Sa-Gla had similar safety and tolerability, including anti-insulin antibody responses, hypoglycemia, body weight, and adverse events.

\section{Conclusions}

Both insulins Mk-Gla and Sa-Gla demonstrated similar safety and effectiveness over 52 weeks in people with T1DM.

\section{Anti-insulin antibodies and adverse events with biosimilar insulin lispro compared with Humalog insulin lispro in people with diabetes}

Home $P^{1}$, Derwahl $K M^{2}$, Ziemen $M^{3}$, Wernicke-Panten $K^{2}$, Pierre $S^{4}$, Kirchhein $Y^{3}$, Garg $S K^{5}$

${ }^{1}$ Institute for Cellular Medicine, Newcastle University, Newcastle upon Tyne, UK; ${ }^{2}$ Institut für Klinische Forschung und Entwicklung, Berlin, Germany; ${ }^{3}$ Sanofi-Aventis Deutschland, Frankfurt, Germany; ${ }^{4}$ Sanofi, Paris, France; ${ }^{5}$ Barbara Davis Center for Diabetes, University of Colorado Denver, Aurora, $\mathrm{CO}$

Diabetes Technol Ther 2018; 20: 160-170 


\section{Background}

SAR342434 (SAR-Lis) is a biosimilar (follow-on) of insulin lispro (Humalog ${ }^{\circledR}$; Ly-Lis). Two randomized, controlled, open-label, parallel-group, phase 3 studies were conducted to compare the efficacy and safety of SAR-Lis and Ly-Lis in combination with insulin glargine (Lantus). SORELLA 1 was a 12-month study in 507 people with T1DM; SORELLA 2 was a 6-month study in 505 people with T2DM. This study presents the impact of anti-insulin antibodies (AIAs) to SAR-Lis and Ly-Lis on safety and glycemic control.

\section{Methods}

AIA were measured regularly throughout both studies at a centralized laboratory blinded to treatment groups and using a drug-specific AIA assay. The AIA status (positive or negative); AIA titers; and cross-reactivity to human insulin, insulin glargine, and insulin glargine metabolite M1 were analyzed. The potential effect of AIA on safety, particularly as related to hypersensitivity reactions, hypoglycemia, and treatment-emergent adverse events, as well as on glycemic control (HbA1c, insulin dose), was evaluated.

\section{Results}

AIA-positive status at baseline was similar for the two insulins but higher in T1DM than in T2DM. In both studies, the percentage of people newly developing AIA in the two treatment groups or having a $\geq 4$-fold increase in AIA titers did not differ. No relationship was observed between maximum individual AIA titers and change in $\mathrm{HbA} 1 \mathrm{c}$ or insulin dose, hypoglycemia, or hypersensitivity reactions or between efficacy/safety measures and subgroups by presence or absence of treatment-emergent AIA. Hypersensitivity events and events adjudicated as allergic reactions were few and did not differ between the two groups.

\section{Conclusions}

Insulin lispro SAR342434 (SAR-Lis) and the originator insulin lispro (Ly-Lis) had a similar immunogenicity profile in people with T1DM or T2DM.

\section{Comments}

As briefly mentioned in the introduction, the cost of insulin in the United States has skyrocketed in the past decade (8- to 10 -fold increase). Thus, many pharmaceutical companies are focusing on making biosimilar or follow-on insulins to make it more affordable for patients requiring insulin. The studies on a rapid-acting insulin lispro (biosimilar lispro Europe, follow-on lispro U.S.) have been shown to be equally efficacious as the original insulin lispro. The HbAlc drop and insulin dose changes were not significant for both 26- and 52-week studies. As mandated by the FDA, all follow-on insulins or other biologics must be evaluated for at least 1 year to make sure that the compounds are not more antigenic. The studies reported on biosimilar lispro were no more antigenic than the original insulin lispro. Biosimilar insulin glargine has also been developed by two different companies and has shown similar efficacy to the original insulin glargine. Both have been marketed for use in the United States as well as Western Europe.

\section{Analysis of retinal layer thickness in diabetic macular oedema treated with ranibizumab or triamcinolone}

Prager $S G^{l}$, Lammer $J^{l}$, Mitsch $C^{l}$, Hafner $J^{1}$, Pemp $B^{l}$, Scholda $C^{1}$, Kundi $M^{2}$, Schmidt-Erfurth $U^{1}$, Kriechbaum $K^{1}$

${ }^{I}$ Department of Ophthalmology and Optometry, Medical University Vienna, Vienna, Austria; ${ }^{2}$ Center of Public Health, Medical University Vienna, Vienna, Austria

Acta Ophthalmol 2018; 96: e195-e200

\section{Purpose}

Spectral-domain optical coherence tomography (SDOCT) images were recorded during the 1-year follow-up examinations of patients treated for diabetic macula edema (DME) in order to evaluate in detail all changes in retinal layer thickness.

\section{Methods}

Patients with DME were randomized to receive treatment as needed with either $0.5 \mathrm{mg}$ ranibizumab or $8 \mathrm{mg}$ triamcinolone. A post hoc analysis of changes in retinal layer thickness was conducted using the automated layer segmentation of SD-OCT images in eyes that received the randomly assigned treatments. Seven retinal layers were segmented in 49 scans that covered $20^{\circ} \times 20^{\circ}$ area of the macula at baseline and after 12 months of treatment. Changes in the thickness of individual layers were correlated with visual acuity and compared between treatment groups.

\section{Results}

A total of 25 patients with DME (mean age $60.2 \pm 15.1$ years; $n=7$ female) were evaluated. Over 12 months, decreased thickness of retinal nerve fiber layer was associated with increased visual acuity $(\mathrm{r}>0.54 ; P<0.05)$. Decreased thickness in ganglion cell layer (GCL) and GCL + IPL (intensed pulse light) pooled for nasal Early Treatment of Diabetic Retinopathy Study (ETDRS) subfields correlated with VA as follows: ranibizumab $r=0.74$ (GCL) and $r=0.63$ $(\mathrm{GCL}+\mathrm{IPL})$; and triamcinolone $r=0.45(\mathrm{GCL})$ and $r=0.46$ $(\mathrm{GCL}+\mathrm{IPL})$.

\section{Conclusions}

In DME therapy, reduction in retinal nerve fiber layer thickness may have a significant impact on retinal function, unrelated to the pharmacological treatment type that is used. Precise morphologic quantification of neurosensory layers provided by SD OCT offers new insight into pathology and therapeutic targets for diseases and conditions such as DME.

\section{Treating diabetic macular oedema (DMO): real world UK clinical outcomes for the $0.19 \mathrm{mg}$ Fluocinolone Acetonide intravitreal implant (lluvien $^{\mathrm{TM}}$ ) at 2 years}

Fusi-Rubiano $W^{1,2}$, Mukherjee $C^{1,2}$, Lane $M^{1,2}$, Tsaloumas $M D^{1}$, Glover $N^{l}$, Kidess $A^{1}$, Denniston $A K^{1,3}$, Palmer $H E^{1}$, Manna $A^{1}$, Morjaria $R^{1,2}$ 
${ }^{1}$ Ophthalmology Department, Queen Elizabeth Hospital Birmingham, University Hospitals Birmingham NHSFT, Birmingham, UK; ${ }^{2}$ Sandwell and West Birmingham NHS Trust, Birmingham, UK; ${ }^{3}$ Academic Unit of Ophthalmology, Institute of Inflammation and Ageing, University of Birmingham, Birmingham, $U K$

BMC Ophthalmol 2018; 18: 62

\section{Background}

Visual function and structural improvements were compared in pseudophakic eyes with diabetic macular edema (DMO) that had undergone treatment with the $0.19 \mathrm{mg}$ fluocinolone acetonide (FAc) intravitreal implant (Iluvien) in a "real-world" setting.

\section{Methods}

A single-center retrospective evaluation was conducted in patients whose DMO was unresponsive to conventional treatment and was therefore treated using the FAc implant according to UK guidelines. Best corrected visual acuity (BCVA) was the primary efficacy endpoint; OCT evaluations of the macula central retinal and peak macular thickness collected at annual time points constituted the secondary endpoints. The primary safety endpoint was new rise in intraocular pressure (IOP) $>27 \mathrm{mmHg}$, or glaucoma surgery. Patients with $<1$ year of follow-up were excluded from the study.

\section{Results}

Twenty-nine eyes were included in the study, with a mean (SD) follow-up of 792 (270) days. At all timepoints, improvement in BCVA and reduction in macular edema was recorded. Mean improvement in BCVA from baseline was a letter score of 6 on the ETDRS acuity test at year $1(\mathrm{n}=29)$, 6.5 at year $2(n=22)$, and 11 at year $3(n=6)$. Mean central retinal thickness was 451 microns at baseline; 337 microns at year $1 ; 342$ microns at year 2 ; and 314 microns at year 3 . Postimplant IOP-lowering drops were required in two eyes. Over the course of the 3-year study, 18 eyes required supplementary treatment for DMO persistence or recurrence; mean time to supplementary treatment was 12 months.

\section{Conclusion}

This real-world evaluation provides further evidence that the $0.19 \mathrm{mg}$ FAc implant is safe and effective for treating patients with DMO and that it can provide sustained benefit for patients with previously refractory disease.

\section{Comments}

Vision loss due to proliferative diabetic retinopathy and/ or diabetic macular edema continues to be a major health hazard for patients with long-standing diabetes. The first study reported the use of a ranibizumab with triamcinolone combination, and the second study evaluated the role of the Fluocinolone Acetonide intravitreal implant.

The two preceding manuscripts highlight the role of steroids in reducing the progression of vision loss, especially retinal nerve fiber layer thickness and diabetic macular edema, especially with previous refractory disease.

\section{Overall Conclusions}

There is abundant research on new SGLT1 and SGLT2 inhibitors, conducted with the hope of finding therapies that are effective at maintaining optimal glycaemia while avoiding DKA and hypoglycemia. Their cardiovascular safety profile has been well documented in different cardiovascular outcome trials in type 2 diabetes. Dapagliflozin and sotagliflozin show some promise in adjunctive therapy with insulin for patients with T1D, yet it is important to understand how these drugs still increase one's risk for DKA. Although these new therapies are proving to reduce weight and may even decrease the risk of hypoglycemia and help stabilize glucose levels, it is necessary to continue to educate patients on behavioral lifestyle changes as well (i.e., diet and exercise) and find appropriate ways to reduce the risk of DKA.

New biosimilar and follow-on insulins can potentially lower the cost burden of insulin-requiring patients with diabetes. For insulin-dependent patients, it is important to make sure that new biosimilar insulins are not more antigenic, while maintaining efficacy, which is what was found for these new insulin analogues.

In order to help prevent other deleterious effects of longterm diabetes, newer ophthalmologic therapies were also explored in this article. New and effective steroid therapies alone or in combination with ranibizumab for macular edema and proliferative diabetic retinopathy demonstrate better vision outcomes for patients with diabetes.

Though no single therapy is a cure-all for diabetes, various combinations of therapies can greatly aid in the management of this complex disease.

\section{Author Disclosure Statement}

No competing financial interests exist. 